\title{
EDITORIAL
}

\section{A message for 2015}

Journal of Human Genetics (2015) 60, 109-111; doi:10.1038/jhg.2015.9

$\mathrm{O}$ ne year has passed since I started as the Editor-in-Chief for Journal of Human Genetics $(J H G)$. I tried my best to maintain and to improve the scientific quality of published articles in JHG. Actually the 2013 journal impact factor is improving (2.526). Without the continuous and tremendous support by all the Associated Editors, Editorial Board members and reviewers, such wonderful success in $J H G$ would never have been achieved. I really appreciate their great contribution. I believe that $J H G$ will truly become the leading human genetics journal in the world.

Last year, we experienced a special event. Human Genome Variation $(H G V)$, which is an online open access journal with accompanying database, was newly launched as a new sister journal of JHG. The Editor-in-Chief for $H G V$ is Professor Katsushi Tokunaga who was the former Editor-in-Chief for JHG. One of the important missions of $H G V$ is publishing human mutation data from all over the world together with the easily searchable database of such mutations. JHG and $H G V$ have their own Associate Editors, but completely share the Editorial Board members. JHG seeks more scientific novelty and HGV accepts more mutations from different ethnic groups. Both journals

Alessandro Achilli

Margaret Adam

Kiwamu Akagi

Tomoya O Akama

Isabel Alves

Ravindran Ankathil

Masashi Aoki

Masami Arai

Takahiro Arima

Takuro Arimura

Noritaka Ariyoshi

Koichiro Asano

Eric Austin

Noriyuki Azuma

Shabeesh Balan

David J Balding

Siddharth Banka

Chiara Barbieri

Timothy Barrett

Chiara Batini

Salma Ben Salem

Hathaichanoke Boonyarit

Protiti Bose
Chad A Bousman

Wojciech Branicki

Pedro Brites

Wesley Brook

David John Bunyan

Francesc Calafell

F David Carmona

Viktor Cerny

Pei-Chieng Cha

Nicolas Chassaing

Gyaneshwer Chaubey

Chalisa Louicharoen

Cheepsunthorn

Feng Chen

Jen-hwa Chu

Valrie Cormier-Daire

Sonia Davila

Simon Edvardson

Thore Egeland

Phillip Endicott

Hideki Enokida

Yaniv Erlich

Muhammad Farooq

enhance and promote human genetic researches under the guidance of the Japan Society of Human Genetics.

Finally, I would happily announce that 2015 is a special year for JHG: 60 years anniversary. We are now planning the special scientific sections in the next 12 months for celebrating the anniversary. We look forward to seeing exciting special sections and of course receiving wonderful manuscripts for $J H G$ from all over the world.

\author{
Naomichi Matsumoto \\ Editor-in-Chief \\ Journal of Human Genetics
}

\section{ACKNOWLEDGMENTS}

The Editor-in-Chief, on behalf of the JHG Editorial Board and the Japan Society of Human Genetics, gratefully acknowledges the generous support from peer reviewers in carefully assessing manuscripts under consideration. The following individuals provided reviews of manuscripts submitted to the $J H G$ in 2014:

Rui Feng

David FitzPatrick

Florentia Fostira

Akihiro Fujimoto

Ken-ichi Fujita

Maki Fukami

Koya Fukunaga

Yoshimitsu Fukushima

Manabu Funayama

Hiroshi Furukawa

Tetsushi Furukawa

RIchard Gibbons

Santhosh Girirajan

Jun Gojobori

Antonio Gonzalez-Martfn

Jun Goto

Yu-ichi Goto

Minxin Guan

Renzo Guerrini

Leonor Gusmao

Hiromichi Hamada

Naoki Harada

Kanae Hasegawa
Tomonobu Hasegawa

Ryota Hashimoto

Ken-ichirou Hata

Shin Hayashi

Yukiko K Hayashi

Meian he

Robert Hill

Shinichi Hirose

Tomomitsu Hirota

Akitoyo Hishimoto

Yuki Hitomi

Nobuyuki Hizawa

Reiko Horikawa

Shin-ichi Horike

Kikuko Hotta

Yoshihiro Hotta

Yue-Qing $\mathrm{Hu}$

Zhibin $\mathrm{Hu}$

Fen Huang

Kazumoto Iijima

Masashi Ikeda

Toshiro Ikeda

Yoshio Ikeda 
Minako Imamura

Issei Imoto

Hidehito Inagaki

Ituro Inoue

Jun Inoue

Ken Inoue

Naoko Ishihara

Kinya Ishikawa

Kotaro Ishikawa

Taisuke Ishikawa

Minoru Isomura

Hidemi Ito

Kazuya Iwamoto

Atsushi Iwata

Masayo Kagami

Yoichiro Kamatani

Kei Kamide

Tomohiro Kamoda

Tadashi Kaname

Tetsufumi Kanazawa

Piranit Kantaputra

Takema Kato

Hideshi Kawakami

Hiroshi Kawame

Aya Kawasaki

Satoshi Kawasaki

Chiea Chuen Khor

Jeong Hyun Kim

Akinori Kimura

En Kimura

Ryosuke Kimura

Takashi Kitano

Kazuma Kiyotani

Peter Knijff

Gen Kobashi

Yuta Kochi

Takashi Kohno

Ikuta Koichi

Takahiro Kojima

Naoshi Kondo

Yutaka Kondo

Tatsuro Kondoh

Shinji Kosugi

Ikuyo Kou

Takeo Kubota

Hiroyuki Kugoh

Akihiro Kume

Shoen Kume

Hiroshi Kunugi

Shigeo Kure

Yoko Kuroki

Kenji Kurosawa

Naohiro Kurotaki

Siegfried Labeit

Michael Lee

Ming-Ta Michael Lee

Eric Lewitus

Guojun Li

Ming Li

Angela LIn

Hui-Yi Lin
Shili Lin

Jiachun $\mathrm{Lu}$

Xiong-jian Luo

Xiongjian Luo

James Lupski

Sallyann Lynch

Sai Ma

Shiro Maeda

Surakameth Mahasirimongkol

Boris A Malyarchuk

John A Martignetti

Hirofumi Maruyama

Tohru Masui

Mitsuo Masuno

Lisa Matisoo-Smith

Keiko Matsubara

Yoichi Matsubara

Hisako Matsumoto

Tatsuo Matsunaga

Mari Matsuo

Roberto Mendoza

Daiki Miki

Koshi Mimori

Kanshi Minamitani

Hiroyuki Mishima

Satomi Mitsuhashi

Jun Mitsui

Kiyonori Miura

Hiroko Miyadera

Taku Miyagawa

Etsuko Miyagi

Hidehiko Miyake

Noriko Miyake

Nobuhisa Mizuki

Ikuko Mizuta

Cara Monroe

Stephen Montgomery

Hiroko Morisaki

Takayuki Morisaki

Shinichi Moriwaki

Jean Muller

Stefan Mundlos

Yoshinori Murakami

Masaaki Muramatsu

Taisei Mushiroda

Kaori Muto

Andrea Nemeth

Yoshitaka Nagai

Satoshi nagayama

Izumi Naka

Akinori Nakamura

Katsunori Nakamura

Takahiro Nakamura

Kazuhiro Nakayama

Eiji Nanba

Irene Netchine

Yen-Hsuan Ni

Wei Nie

Nao Nishida

Gen Nishimura

Ichizo Nishino
Jo Nishino

Hisahide Nishio

Shin-ya Nishio

Emiko Noguchi

Yoshihiro Noguchi

Kandai Nozu

Hironao Numabe

Hidenori Ochi

Atsushi Ogawa

Takuya Ogawa

Jun Ohashi

Toya Ohashi

Miki Ohira

Tohru Ohta

Tamae Ohye

Yukinori Okada

Yukinori Okada

Nobuhiko Okamoto

Yasushi Okazaki

Torayuki Okuyama

Osamu Onodera

Yoshihiro Onouchi

Yoshihiro Onouchi

Hiroshi Onuma

Kouichi Ozaki

Takeshi Ozeki

Min-Sheng Peng

Min-Sheng Peng

Thomas Prior

Enkhsaikhan Purevjav

Francesco Ramirez

Lanka Ranaweera

H Rennert

Saima Riazuddin

Antonia Ribes

Jorge Rocha

Shinji Saito

Yoshiro Saito

Naruya Saitou

Hirotomo Saitsu

Norio Sakai

Osamu Sakamoto

Tohru Sakamoto

Akihiro Sakurai

Daisuke Sakurai

Osamu Samura

Cristina Santos

Masayuki Sasaki

Noriko Sato

Setsu Sawai

Laura Scheinfeldt

Theodore G Schurr

Naohiko Seki

Stephanie Sherman

Hong Shi

Hiroki Shibata

Daichi Shigemizu

Haruo Shimazaki

Keiko Shimojima

Nobuyuki Shimozawa

Yuichi Shiraishi
Mikio Shoji

Sarah Smithson

Myunghyun Sohn

Yuki Kawashima Sonoyama

Metawee Srikummool

Nathan Stitziel

Bhoom Suktitipat

Liangdan Sun

Andrea Superti-Furga

Hideo Suzuki

Yoichi Suzuki

Matthis Synofzik

Yasuharu Tabara

Hayato Tada

Hidetoshi Tahara

Atsushi Tajima

Atsushi Takahashi

Masanori P Takahashi

Yuji Takahashi

Kyoko Takano

Jun Takeda

Yasuhiro Takeshima

Toshihiro Takizawa

Mayumi Tamari

Akemi Tanaka

Fumiaki Tanaka

Hisashi Tanaka

Toshihiro Tanaka

Mariko Taniguchi-Ikeda

Paul Thomas

Iole Tomassini Barbarossa

Draga Toncheva

Po-Nien Tsao

Naoyuki Tsuchiya

Kazuhiro Tsukamoto

Henna Tyynismaa

Shin-ichi Usami

Eric Vallender

Marjo van der Knaap

Mannis van Oven

Alexandre Rezende Vieira

Yskert von Kodolitsch

Takahito Wada

Keiko Wakui

Chaolong Wang

Matthew Warman

Hiroshi Watanabe

Jason A Wilder

Katharina Wimmer

Rongling $\mathrm{Wu}$

Shuhua $\mathrm{Xu}$

Xiangmin $\mathrm{Xu}$

Shigehito Yamada

Takahiro Yamada

Yoshiji Yamada

Takanori Yamagata

Kazuhiro Yamakawa

Ken Yamamoto

Toshiyuki Yamamoto

Keiko Yamazaki

Itaru Yanagihara 
Kazuki Yasuda

Sook Wah Yee

Toshifumi Yokota

Tohru Yorifuji
Ogawa Yoshihiro

Takashi Yoshikado

Koh-ichiro Yoshiura

Xianglin Yuan
Pierre A Zalloua

Andreas Zankl

Wen Zhang

Zhiwu Zhang
Wolfgang Zimmermann

Johannes Zschocke 